\title{
Fish consumption in early pregnancy and congenital gastrointestinal tract atresia in the Japan Environment and Children's Study
}

\author{
Takehiro Michikawa ${ }^{1 *}$, Shin Yamazaki ${ }^{1}$, Masaji Ono ${ }^{1}$, Tatsuo Kuroda ${ }^{2}$, Shoji F. Nakayama ${ }^{1}$, Eiko Suda ${ }^{1}$, \\ Tomohiko Isobe $^{1}$, Miyuki Iwai-Shimada ${ }^{1}$, Yayoi Kobayashi ${ }^{1}$, Kenji Tamura ${ }^{1}$, Junzo Yonemoto ${ }^{1}$, \\ Toshihiro Kawamoto ${ }^{1,3}$, Hiroshi Nitta $^{1}$ and the Japan Environment and Children's Study Group $\dagger$ \\ ${ }^{1}$ Centre for Health and Environmental Risk Research, National Institute for Environmental Studies, 16-2 Onogawa, Tsukuba, \\ Ibaraki 305-8506, Japan \\ ${ }^{2}$ Department of Paediatric Surgery, Keio University School of Medicine, 35 Shinanomachi, Shinjuku-ku, \\ Tokyo 160-8582, Japan \\ ${ }^{3}$ Department of Environmental Health, University of Occupational and Environmental Health, 1-1 Iseigaoka, Yahatanishi-ku, \\ Kitakyushu, Fukuoka 807-8556, Japan
}

(Submitted 18 April 2018 - Final revision received 23 August 2018 - Accepted 5 September 2018 - First published online 29 October 2018 )

\section{Abstract}

Current evidence suggests that the aetiology of congenital gastrointestinal (GI) tract atresia is multifactorial, and not based solely on genetic factors. However, there are no established modifiable risk factors for congenital GI tract atresia. We used data from a Japanese nationwide birth cohort study launched in 2011, and examined whether fish consumption in early pregnancy was associated with congenital GI tract atresia. We analysed data of 89495 women (mean age at delivery $=31.2$ years) who delivered singleton live births without chromosomal anomalies. Based on the results of the FFQ, we estimated the daily intake of fish and $n$ - 3 PUFA consumption in early pregnancy. We defined a composite outcome (oesophageal atresia, duodenal atresia, jejunoileal atresia and/or anorectal malformation) as congenital GI tract atresia. In this population, median fish intake was $31.9 \mathrm{~g} / \mathrm{d}$, and seventy-four cases of congenital GI tract atresia were identified. Fish consumption in early pregnancy was inversely associated with the composite outcome (multivariable-adjusted OR for the high $v$. low consumption category $=0.5,95 \% \mathrm{CI} 0.3,1 \cdot 0$ ). For all the specific types of atresia, decreased OR were observed in the high consumption category, although not statistically significant. Reduced atresia occurrence was observed even beyond the US Food and Drug Administration's recommended consumption of no more than $340 \mathrm{~g} /$ week. Also, $n$-3 PUFA-rich fish and $n$-3 PUFA consumptions tended to be inversely associated with atresia. Fish consumption in early pregnancy may be a preventive factor for congenital GI tract atresia.

Key words: Fish consumption: Oesophageal atresia: Intestinal atresia: Anorectal malformation: Birth cohorts

Oesophageal atresia, small intestinal atresia (duodenal and jejunoileal atresia), and anorectal malformation, which we collectively defined as congenital gastrointestinal (GI) tract atresia in this study, are relatively common congenital anomalies. In the 2014 annual report of the International Centre on Birth Defects, the mean frequencies, as reported by twenty-seven monitoring systems, were 2.8 cases (per 10000 births) for oesophageal atresia, $2 \cdot 7$ cases for small intestinal atresia and 3.8 cases for anorectal malformation $^{(1)}$. In Japan, congenital GI tract atresia was a leading cause of neonatal surgical diseases in $2013^{(2)}$. Due to medical advances, the mortality rate of children born with GI tract atresia has decreased year by year ${ }^{(3)}$; however, related long-term morbidities, such as feeding gastrostomy or jejunostomy, short bowel syndrome, faecal and/or urinary incontinence, and chronic constipation ${ }^{(4-6)}$, continue to have a significant impact on childhood quality of life. In addition, occurrence of the condition typically increases the psychological and social burden on mothers, as well as on their partners and families due to concern for the child's health. Thus, if modifiable factors leading to primary prevention of congenital GI tract atresia can be discovered, it is good news for both child and maternal health.

The embryonic period ( $4-8$ weeks of gestation) is known as the developmental origin of congenital GI tract atresia. For oesophageal atresia, a failure of invagination of the lateral trachea-oesophageal grooves is suggested ${ }^{(7)}$. Small intestinal

Abbreviations: GI, gastrointestinal; JECS, Japan Environment and Children's Study.

* Corresponding author: T. Michikawa, email tmichikawa@nies.go.jp

$\dagger$ The study group members are listed in the Appendix. 
atresia is believed to occur as a result of unsuccessful recanalisation of the bowel, though the probable cause of some cases, in particular jejunoileal atresia, may be a late vascular accident ${ }^{(8)}$. Impaired process in the urorectal septum is likely to lead to the development of anorectal malformation $^{(9)}$. However, the aetiology of congenital GI tract atresia (the cause of the embryologic abnormality) is still unclear. Although the importance of genetic background to the aetiology has been generally accepted ${ }^{(8,10,11)}$, we know that its development is not caused solely by genetic factors; exogenous factors also contribute to the pathogenesis of GI tract atresia.

As far as we know, there are no established modifiable risk factors (and therefore preventions) for congenital GI tract atresia; in contrast, for example, to the administration of folic acid to prevent neural tube defects ${ }^{(12)}$. Past studies have revealed an association between prepregnancy diabetes or gestational diabetes and obesity or overweightness with GI tract atresia, in particular oesophageal atresia and anorectal malformation ${ }^{(13-19)}$. There is a pathological link between diabetes and obesity, leading to an increase in inflammation and oxidative stress in utero ${ }^{(20)}$. Thus, given the possibility that diabetes and/or obesity have a role in the development of GI tract atresia, we were interested in diets that form an essential part of human life, and related potentially modifiable factors. We focused on fish because fish consumption is considered to be associated with a reduced risk of obesity and diabetes; and $n$-3 PUFA contained in fish oil ${ }^{(21)}$, which are transported across the placenta ${ }^{(22)}$, are likely to have beneficial effects on inflammation and oxidative stress ${ }^{(23,24)}$. In addition, fish is an important ingredient in the form of Japanese cuisine (washoku) characterised by a well-balanced and healthy diets. We hypothesised that fish consumption would be inversely associated with the occurrence of congenital GI tract atresia.

The aim of this study was to examine the association between fish consumption in early pregnancy (the embryonic period) and congenital GI tract atresia.

\section{Methods}

\section{Study participants}

The concept and design of the Japan Environment and Children's Study (JECS), an ongoing nationwide birth cohort study, are described in detail elsewhere ${ }^{(25)}$. Briefly, we recruited women as in early pregnancy as possible in fifteen regional centres located throughout Japan, and registered 103099 pregnancies between 2011 and $2014^{(26)}$. After exclusion of 2321 with no subsequent delivery record, the remaining 100778 pregnancies involved 101779 foetuses, and resulted in 100148 live births. The selected characteristics of the mothers and children were similar in the JECS and general Japanese population $^{(26)}$. The women's partners (fathers) were approached whenever possible. The distribution of maternal and infant characteristics among the sub-population with participating fathers (approximately 50000) was essentially the same as that of the total population ${ }^{(26)}$. This study was conducted according to the guidelines laid down in the
Declaration of Helsinki and all procedures involving participants were approved by the Japan Ministry of the Environment's Institutional Review Board on Epidemiological Studies (no. 100406001), and the Ethics Committees of all participating institutions. Written informed consent was obtained from all participants.

In this study, we restricted the study participants to 95170 unique mothers (the first JECS pregnancy) among the 100778 pregnancies included women registered multiple times for siblings. From these, as shown in online Supplementary Fig. S1, we selected 89658 mothers who delivered singleton live births, and responded to a FFQ during the first trimester. Then, we excluded 163 participants who delivered children with chromosomal anomalies, including Down syndrome, trisomy 18 or trisomy 13 , in order to exclude the possibility that congenital GI tract atresia was caused by chromosomal factors ${ }^{(10,11,27)}$. In the end, a total of 89495 mothers ( 89495 births) were included in our analysis.

\section{Fish consumption}

Self-administered questionnaires, including FFQ, were distributed twice, first during the first trimester (median fill-in week of gestation $=15$ ), and then again during the second/third trimester (27th week of gestation). In the first FFQ, we asked about usual dietary intake in the preceding year, and in the second, usual intake after awareness of pregnancy. In this study, we mainly used data from the first FFQ as a marker of dietary intake in early pregnancy.

We adopted the FFQ used in the Japan Public Health Centre-based prospective study for the next generation ${ }^{(28)}$. It contained 172 food and beverage items, including twentyone items of fish or shellfish (salted fish, dried fish, canned tuna, salmon or trout, bonito or tuna, Japanese amberjack, cod or flatfish, sea bream, horse mackerel or sardine, saury or mackerel, dried small fish, salted roe, eel, squid, octopus, prawn, clam, pond-snail and three fish paste products (chikuwa, kamaboko and satsuma-age)). The choices of portion size for each food item were: small (50\% less than standard), medium (equal to standard) and large (50\% more than standard). We used nine frequency categories $(<1$ time/month, 1-3 times/month, 1-2 times/week, 3-4 times/ week, 5-6 times/week, 1 time/d, 2-3 times/d, 4-6 times/d and $\geq 7$ times/d). The daily intake of fish consumption was calculated by multiplying the frequency by the standard-equivalent portion size, for each fish item. In addition, $n$-3 PUFA-rich fish consumption was defined from six fish items (salmon or trout, Japanese amberjack, sea bream, horse mackerel or sardine, saury or mackerel and eel). We further calculated the daily intake of $n-3$ PUFA, including DHA, EPA and $\alpha$-linolenic acid, using the Standard Tables of Food Composition in Japan, the fifth revised and enlarged edition 2005 for fatty acids ${ }^{(29)}$. For this FFQ, in 142 Japanese women aged 40-74 years, Spearman's correlation coefficients between intakes, as estimated from the FFQ and the $12 \mathrm{~d}$ weighted food record, were 0.47 for fish and 0.40 for $n-3$ PUFA $^{(28)}$. We did not have information on cooking-method based fish intake. 


\section{Congenital gastrointestinal tract atresia}

In accordance with the JECS in-house standard operating procedures, medical record transcriptions were performed three times by physicians, midwives/nurses and/or research coordinators: first during the first trimester, second after delivery and finally at the first-month health check-up after delivery. The transcription report forms after delivery and at a month after delivery contained a list of congenital anomalies, including oesophageal atresia, small intestinal atresia (duodenal and jejunoileal atresia) and anorectal malformation. As the numbers of each type of atresia were relatively small, we defined a composite outcome (oesophageal atresia, small intestinal atresia and/or anorectal malformation) as congenital GI tract atresia.

\section{Statistical analysis}

We categorised participants into tertiles of fish consumption in early pregnancy. To explore the association of fish consumption with congenital GI tract atresia and each specific type of atresia, we used a logistic regression model, and estimated the OR and $95 \%$ CI of atresia, with the low consumption category as a reference. Initially, we adjusted for maternal age at delivery $(<25,25-29,30-34$ and $\geq 35$ years). Although there are no established risk factors for GI tract atresia, we additionally adjusted for several maternal factors as possible confounding factors that have been reported to be associated with congenital anomalies including atresia, as follows: educational background ( $<13$ and $\geq 13$ years); household income ( $<6$ and $\geq 6$ million Japanese-yen/year); occupation in early pregnancy (administrative, managerial, professional, or engineering, clerical, sales and service, homemaker and others); smoking habits (never smoked, ex-smokers who quit before pregnancy and smokers during early pregnancy); alcohol consumption (never drank, ex-drinkers who quit before pregnancy and drinkers during early pregnancy); BMI before pregnancy $(<18 \cdot 5,18 \cdot 5-24.9$ and $\geq 25 \cdot 0 \mathrm{~kg} / \mathrm{m}^{2}$ ); current history of diabetes or gestational diabetes (no, yes); parity $(0, \geq 1)$; infertility treatment (no, ovulation stimulation or artificial insemination by sperm from husband and assisted reproductive technology $)^{(30)}$; use of folic acid supplement (routine use (defined as $\geq 4$ times/week) and nonroutine use) ${ }^{(31)}$; use of DHA and/or EPA supplements (routine use (defined as $\geq 4$ times/week) and non-routine use), and vegetable intake as a marker of healthy dietary habit. For the sub-population with participating fathers, we adjusted for paternal age at delivery $(<25,25-29, \quad 30-34$ and $\geq 35$ years) ${ }^{(32)}$. Linear trends in the association were assessed by assignment of ordinal variables for the fish consumption tertile.

To examine the robustness of the association between fish consumption in early pregnancy and congenital GI tract atresia, we performed several subgroup and sensitivity analyses. First, we examined the association for cases of congenital GI tract atresia alone, after excluding cases with other major congenital anomalies, because genetic factors rather than exogenous factors might contribute to the occurrence of multiple anomalies. In this study, with reference to the US National Birth Defects Prevention Network ${ }^{(33)}$ and the surveillance manual of World
Health Organization $^{(34)}$, major anomalies included anencephaly, spina bifida, encephalocele, microphthalmia, cleft palate, cleft lip (with or without cleft palate), congenital heart diseases (not including patent ductus arteriosus), gastroschisis, omphalocele, diaphragmatic hernia, hypospadias, reduction defects of the upper and/or lower limbs, and the specific types of congenital GI tract atresia. Second, we excluded routine users of DHA and/or EPA supplements. Third, we excluded women with severe morning sickness. Fourthly, we restricted to participants without diabetes, gestational diabetes, obesity or overweightness (BMI $<25.0 \mathrm{~kg} / \mathrm{m}^{2}$ ). Finally, we defined fish consumption to exclude one item, bonito or tuna, because tuna contains relatively high levels of methylmercury ${ }^{(35)}$.

Subsequently, based on the advice about eating fish to pregnant women from US Food and Drug Administration (FDA) (not exceed 12 ounces/week (approximately $340 \mathrm{~g} /$ week)) ${ }^{(36)}$, we classified participants into three groups with regard to fish consumption in early pregnancy, after calculating by multiplying the daily intake (g/d) by $7:<170$ (half of 340 ), 170-340 and >340 g/week. $n$-3 PUFA-rich fish and n-3 PUFA consumption was also examined. In addition, we used data of the second FFQ during the second/third trimester as a marker of dietary intake in mid-late pregnancy, and investigated the association between fish consumption in mid-late pregnancy and congenital GI tract atresia. For this analysis, we included 88488 women who had valid data from the second FFQ and delivered their babies after more than 28 weeks of gestation.

The present study used the dataset jecs-ag-20160424, which was released in June 2016, and revised in October 2016, along with the supplementary dataset jecs-ag-20160424-sp1. All analyses were performed with Stata 14 (StataCorp LP).

\section{Results}

Among 89495 pregnant women (mean age at delivery $=31 \cdot 2$ years), the median fish intake in early pregnancy was $31.9 \mathrm{~g} / \mathrm{d}$. The proportions of women in the following subgroups tended to be higher for the high category of fish consumption: aged 35 years or older, educational background $\geq 13$ years, household income $\geq 6$ million Japanese-yen/year, homemakers, neversmokers, parous women and with partners aged 35 years or older (Table 1). Vegetable intake appeared to increase with increasing fish intake. Among the 89495 births (median gestational age $=39$ week), we identified seventy-four cases of congenital GI tract atresia (online Supplementary Table S1).

We found an inverse association between fish consumption and congenital GI tract atresia (Table 2). The occurrence of congenital GI tract atresia was lower in women in the high than in the low consumption category (multivariable-adjusted $\mathrm{OR}=0.5,95 \% \mathrm{CI} 0 \cdot 3,1 \cdot 0$ ), and this inverse association persisted after adjustment for paternal age at delivery. Decreased OR were observed for all the specific types of atresia in the high consumption category, although not statistically significant. Our results did not essentially change in several subgroup analyses (Table 3). Also, the results of the sensitivity analysis based on fish consumption excluding fish containing relatively high methylmercury showed a similar pattern. Moreover, reduced 
Table 1. Baseline characteristics of 89495 pregnant women, Japan Environment and Children's Study (2011-2014)

(Percentages and median values)

\begin{tabular}{|c|c|c|c|c|}
\hline & \multirow[b]{3}{*}{ Number of women* } & \multicolumn{3}{|c|}{ Tertile of fish consumption in early pregnancy } \\
\hline & & \multirow{2}{*}{$\frac{\operatorname{Low}(n 29831)}{\%}$} & \multirow{2}{*}{$\frac{\text { Middle }(n \text { 29832) }}{\%}$} & \multirow{2}{*}{$\frac{\text { High }(n 29832}{\%}$} \\
\hline & & & & \\
\hline Daily intake of fish (g/d) & 89495 & & & \\
\hline Median & & 12.9 & 31.9 & $56 \cdot 5$ \\
\hline \multicolumn{5}{|l|}{ Age at delivery (years) } \\
\hline$<25$ & 8651 & $13 \cdot 3$ & $8 \cdot 4$ & $7 \cdot 3$ \\
\hline $25-29$ & 24588 & $30 \cdot 2$ & $27 \cdot 0$ & $25 \cdot 2$ \\
\hline $30-34$ & 31754 & $33 \cdot 2$ & $36 \cdot 4$ & $36 \cdot 8$ \\
\hline$\geq 35$ & 24502 & $23 \cdot 2$ & $28 \cdot 2$ & $30 \cdot 7$ \\
\hline \multicolumn{5}{|l|}{ Educational background (years) } \\
\hline$<13$ & 31628 & $41 \cdot 1$ & $34 \cdot 2$ & $32 \cdot 3$ \\
\hline$\geq 13$ & 56610 & $58 \cdot 9$ & $65 \cdot 8$ & $67 \cdot 8$ \\
\hline \multicolumn{5}{|l|}{ Household income (million Japanese-yen/year) } \\
\hline$<6$ & 60260 & $76 \cdot 9$ & $72 \cdot 2$ & $70 \cdot 2$ \\
\hline$\geq 6$ & 22235 & $23 \cdot 1$ & $27 \cdot 8$ & $29 \cdot 8$ \\
\hline \multicolumn{5}{|l|}{ Occupation in early pregnancy } \\
\hline Administrative, managerial, professional or engineering & 20613 & $22 \cdot 1$ & 23.5 & $24 \cdot 1$ \\
\hline Clerical & 15255 & $17 \cdot 2$ & $17 \cdot 0$ & $17 \cdot 4$ \\
\hline Sales and service & 19510 & $25 \cdot 1$ & $20 \cdot 7$ & $20 \cdot 2$ \\
\hline Homemaker & 24754 & $24 \cdot 7$ & $29 \cdot 3$ & $29 \cdot 6$ \\
\hline Others & 8691 & $11 \cdot 0$ & $9 \cdot 6$ & 8.8 \\
\hline \multicolumn{5}{|l|}{ Smoking habits } \\
\hline Never smoked & 52124 & 54.9 & $59 \cdot 8$ & $60 \cdot 2$ \\
\hline Ex-smokers who quit before pregnancy & 20923 & $23 \cdot 2$ & 23.5 & $23 \cdot 6$ \\
\hline Smokers during early pregnancy & 16322 & 21.9 & $16 \cdot 7$ & $16 \cdot 2$ \\
\hline \multicolumn{5}{|l|}{ Alcohol consumption } \\
\hline Never drank & 30798 & 35.4 & 34.5 & 33.4 \\
\hline Ex-drinkers who quit before pregnancy & 16697 & $18 \cdot 4$ & $18 \cdot 4$ & $19 \cdot 3$ \\
\hline Drinkers during early pregnancy & 41983 & $46 \cdot 3$ & $47 \cdot 1$ & $47 \cdot 3$ \\
\hline \multicolumn{5}{|l|}{ BMI before pregnancy $\left(\mathrm{kg} / \mathrm{m}^{2}\right)$} \\
\hline$<18.5$ & 14450 & $16 \cdot 4$ & $16 \cdot 2$ & $15 \cdot 8$ \\
\hline $18 \cdot 5-24 \cdot 9$ & 65552 & $73 \cdot 1$ & 73.5 & $73 \cdot 3$ \\
\hline$\geq 25 \cdot 0$ & 9455 & $10 \cdot 5$ & $10 \cdot 2$ & $11 \cdot 0$ \\
\hline \multicolumn{5}{|l|}{ Current history of diabetes or gestational diabetes } \\
\hline No & 86678 & $97 \cdot 2$ & $96 \cdot 9$ & $96 \cdot 5$ \\
\hline Yes & 2817 & $2 \cdot 8$ & $3 \cdot 1$ & 3.5 \\
\hline \multicolumn{5}{|l|}{ Parity } \\
\hline 0 & 39041 & $49 \cdot 2$ & $41 \cdot 6$ & 40.5 \\
\hline$\geq 1$ & 50150 & $50 \cdot 8$ & $58 \cdot 4$ & $59 \cdot 5$ \\
\hline Infertility treatment & & & & \\
\hline No & 83439 & $94 \cdot 1$ & $93 \cdot 1$ & 92.5 \\
\hline Ovulation stimulation or artificial insemination by sperm from husband & 3291 & 3.4 & $3 \cdot 8$ & 3.9 \\
\hline Assisted reproductive technology & 2737 & 2.5 & $3 \cdot 1$ & 3.6 \\
\hline Dietary intake & & & & \\
\hline Energy $(\mathrm{kJ} / \mathrm{d})$ & 89495 & & & \\
\hline Median & & 6764 & 7147 & 7257 \\
\hline Vegetables $(\mathrm{g} / \mathrm{d})$ & 89495 & & & \\
\hline Median & & $133 \cdot 2$ & $159 \cdot 5$ & $177 \cdot 0$ \\
\hline Routine use of supplements & & & & \\
\hline Folic acid & 23978 & $25 \cdot 7$ & $27 \cdot 9$ & $27 \cdot 8$ \\
\hline DHA and/or EPA & 1465 & 1.7 & 1.5 & $1 \cdot 8$ \\
\hline Morning sickness & & & & \\
\hline No or not severe & 78600 & $89 \cdot 0$ & 89.6 & $88 \cdot 6$ \\
\hline Severe & 9679 & $11 \cdot 1$ & $10 \cdot 4$ & 11.5 \\
\hline Gestational age (weeks) & 89495 & & & \\
\hline Median & & 39 & 39 & 39 \\
\hline Preterm (<37 weeks) & 4151 & $4 \cdot 7$ & 4.6 & $4 \cdot 7$ \\
\hline Infant sex & & & & \\
\hline Boy & 45945 & 51.4 & $51 \cdot 4$ & $51 \cdot 3$ \\
\hline Girl & 43542 & $48 \cdot 6$ & $48 \cdot 6$ & $48 \cdot 7$ \\
\hline Paternal age at delivery (years) & & & & \\
\hline$<25$ & 2981 & $8 \cdot 8$ & $5 \cdot 9$ & 4.5 \\
\hline $25-29$ & 10588 & $27 \cdot 0$ & $21 \cdot 3$ & $20 \cdot 1$ \\
\hline $30-34$ & 15350 & 31.9 & $34 \cdot 2$ & $33 \cdot 1$ \\
\hline$\geq 35$ & 17536 & 32.4 & 38.6 & $42 \cdot 2$ \\
\hline
\end{tabular}

* Subgroup numbers do not total to overall number due to missing data. 
Table 2. Fish consumption in early pregnancy and congenital gastrointestinal tract atresia (Odds ratios and $95 \%$ confidence intervals; median values and numbers)

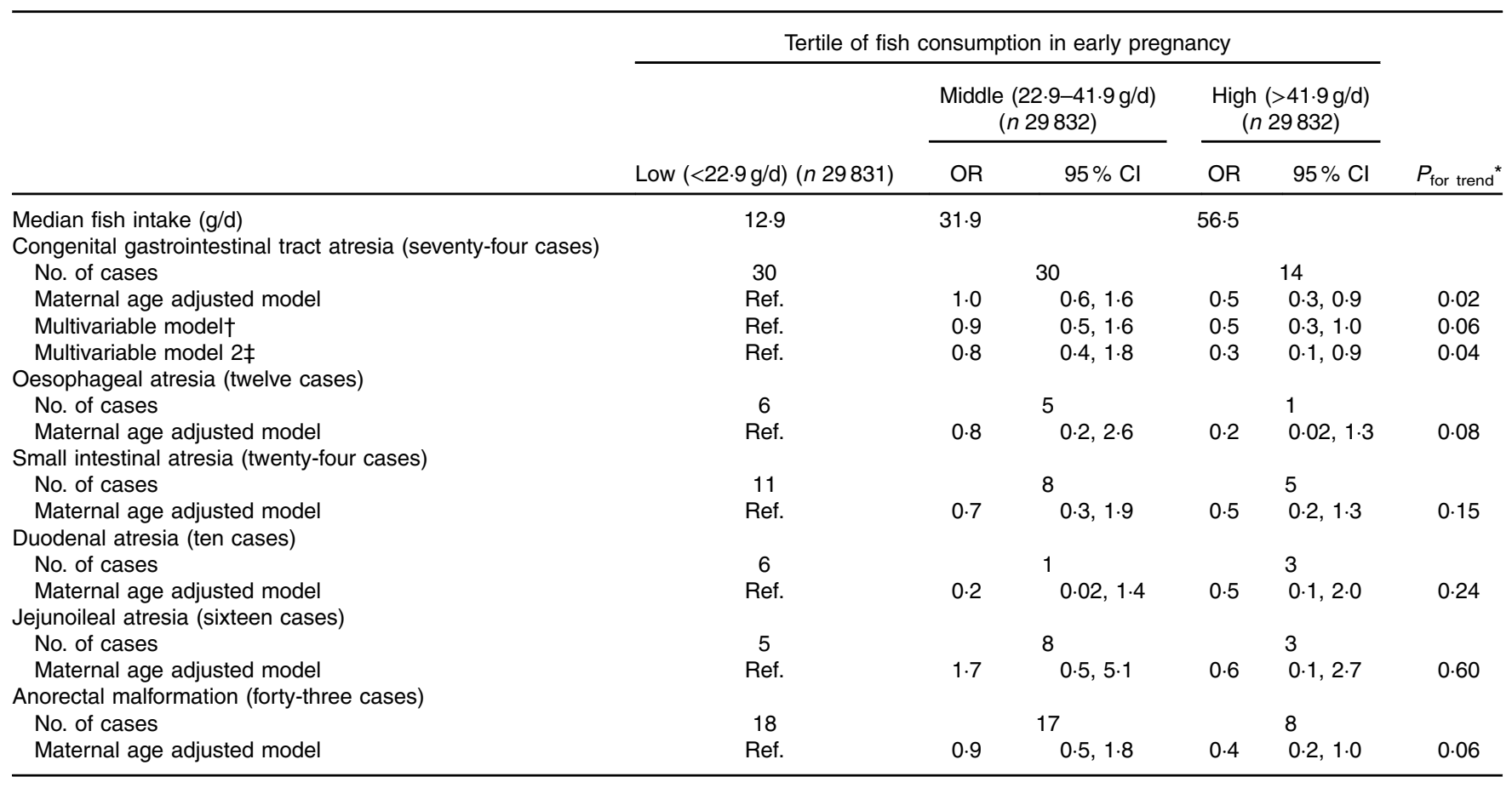

Ref., reference.

* Linear trends were assessed by assignment of ordinal variables for fish consumption tertile.

† Adjusted for maternal age at delivery, educational background, household income, occupation in early pregnancy, smoking habits, alcohol consumption, BMI before pregnancy, current history of diabetes or gestational diabetes, parity, infertility treatment, use of folic acid supplement, use of DHA and/or EPA supplements and vegetable consumption in early pregnancy.

$\ddagger$ Additionally adjusted for paternal age at delivery $(n 46455)$.

atresia occurrence was observed even beyond the US FDA's recommended consumption of no more than $340 \mathrm{~g} /$ week (multivariable-adjusted OR for $>340 v .<170 \mathrm{~g} /$ week $=0.4,95 \%$ CI $0.2,0 \cdot 9$ ) (online Supplementary Table S2).

We observed that $n$-3 PUFA-rich fish consumption in early pregnancy was inversely associated with congenital GI tract atresia (multivariable-adjusted OR for high $v$. low consumption $=0.5,95 \%$ CI $0.3,1.0$ ) (Table 4). However, there was no association with $n$-3 PUFA consumption, although the OR point estimate was less than unity (multivariable-adjusted $\mathrm{OR}=0.7$, $95 \%$ CI $0 \cdot 4,1 \cdot 4)$.

With respect to fish consumption in mid-late pregnancy, Spearman's correlation coefficient for fish intake in early pregnancy was $0 \cdot 52$. The multivariable-adjusted OR for congenital GI tract atresia were $0.5(95 \%$ CI $0.3,1.0)$ in the high tertile (v. low tertile) (online Supplementary Table S3). Neither $n$-3 PUFA-rich fish consumption nor $n-3$ PUFA consumption in midlate pregnancy were not clearly associated with GI tract atresia (online Supplementary Table S4).

\section{Discussion}

In a birth cohort consisting of members of the general Japanese population who traditionally eat fish, we examined whether fish consumption was associated with congenital GI tract atresia diagnosed by the first month after birth. As far as we know, this is the first study to find the inverse association between fish consumption in early pregnancy and GI tract atresia.

Fish consumption in early pregnancy was associated with a decreased risk of congenital GI tract atresia. Moreover, reduced atresia occurrence was observed even beyond the fish consumption target recommended by the US FDA to avoid exposure to methylmercury in utero $^{(36)}$. Even when we used fish consumption excluding fish containing a relatively high level of methylmercury, regarding which the Japan Ministry of Health, Labour and Welfare announced a recommended intake for pregnant women ${ }^{(35)}$, a similar inverse association was demonstrated. Another remarkable point is that $n$-3 PUFA-rich fish consumption was inversely associated with GI tract atresia, suggesting that $n-3$ PUFA from fish oil (i.e. DHA and EPA), which are transported across the placenta ${ }^{(22)}$, appear to have beneficial effects for atresia prevention. On the other hand, we found no association for total $n-3$ PUFA consumption, though somewhat decreased odds of occurrence were noted in this case. This is not surprising, because approximately $60 \%$ of $n-3$ PUFA intake in Japan contains $\alpha$-linolenic acid (mainly from vegetables) $)^{(37)}$, which synthesises only a small amount of $\mathrm{EPA}^{(24)}$. In excluding routine users of DHA/EPA supplements, the present study revealed the possibility that dietary DHA/EPA intake influenced atresia occurrence. From the viewpoint of prevention of congenital GI tract atresia, our results suggest that an increase in varied fish consumption, with careful attention to avoid fish containing a relatively level of high methylmercury, is 
Table 3. Subgroup and sensitivity analysis of the association between fish consumption in early pregnancy and congenital gastrointestinal tract atresia (Odds ratios and $95 \%$ confidence intervals; median values and numbers)

\begin{tabular}{|c|c|c|c|c|c|c|}
\hline & \multicolumn{5}{|c|}{ Tertile of fish consumption in early pregnancy } & \multirow[b]{3}{*}{$P_{\text {for trend }}{ }^{*}$} \\
\hline & \multirow[b]{2}{*}{ Low } & \multicolumn{2}{|r|}{ Middle } & \multicolumn{2}{|c|}{ High } & \\
\hline & & OR & $95 \% \mathrm{Cl}$ & OR & $95 \% \mathrm{Cl}$ & \\
\hline \multicolumn{7}{|l|}{ Total fish consumption } \\
\hline Median intake $(\mathrm{g} / \mathrm{d})$ & 12.9 & 31.9 & & 56.5 & & \\
\hline \multicolumn{7}{|c|}{ Isolated cases of congenital gastrointestinal tract atresia (cases without other major anomalies) } \\
\hline No. of women & 29823 & \multicolumn{2}{|r|}{29825} & \multicolumn{2}{|c|}{29830} & \\
\hline No. of cases & 22 & \multicolumn{2}{|r|}{23} & \multicolumn{2}{|c|}{12} & \\
\hline Multivariable model† & Ref. & 1.0 & $0.5,1.9$ & 0.6 & $0.3,1 \cdot 2$ & 0.18 \\
\hline \multicolumn{7}{|c|}{ Excluding routine users of DHA and/or EPA supplements } \\
\hline No. of women & 28711 & \multicolumn{2}{|r|}{28866} & \multicolumn{2}{|c|}{28778} & \\
\hline No. of cases & 28 & \multicolumn{2}{|r|}{30} & \multicolumn{2}{|r|}{14} & \\
\hline Multivariable model† & Ref. & 1.0 & $0.6,1.8$ & 0.6 & $0.3,1 \cdot 1$ & 0.10 \\
\hline \multicolumn{7}{|c|}{ Excluding participants with severe morning sickness } \\
\hline No. of women & 26588 & \multicolumn{2}{|r|}{26771} & \multicolumn{2}{|c|}{26457} & \\
\hline No. of cases & 27 & \multicolumn{2}{|r|}{30} & \multicolumn{2}{|r|}{12} & \\
\hline Multivariable model† & Ref. & 0.9 & $0.5,1.6$ & 0.4 & $0.2,0.9$ & 0.03 \\
\hline \multicolumn{7}{|c|}{ Restricting to participants without diabetes or gestational diabetes } \\
\hline No. of women & 28988 & \multicolumn{2}{|r|}{28910} & \multicolumn{2}{|c|}{28780} & \\
\hline No. of cases & 29 & \multicolumn{2}{|r|}{29} & \multicolumn{2}{|c|}{14} & \\
\hline Multivariable model† & Ref. & 0.9 & $0.5,1.6$ & 0.5 & $0.3,1.0$ & 0.06 \\
\hline \multicolumn{7}{|c|}{ Restricting to participants with a BMI before pregnancy of $<25.0 \mathrm{~kg} / \mathrm{m}^{2}$} \\
\hline No. of women & 26699 & \multicolumn{2}{|r|}{26780} & \multicolumn{2}{|c|}{26561} & \\
\hline No. of cases & 29 & \multicolumn{2}{|r|}{27} & \multicolumn{2}{|r|}{13} & \\
\hline Multivariable model† & Ref. & 0.9 & $0.5,1.6$ & 0.5 & $0.3,1.0$ & 0.05 \\
\hline $\begin{array}{l}\text { Fish consumption exclu } \\
\text { relatively high levels of }\end{array}$ & & & & & & \\
\hline Median intake $(\mathrm{g} / \mathrm{d})$ & 11.5 & $29 \cdot 3$ & & 52.4 & & \\
\hline No. of women & 29831 & & 29832 & & 9832 & \\
\hline No. of cases & 29 & & 29 & & 16 & \\
\hline Multivariable model† & Ref. & 1.0 & $0.6,1.7$ & 0.6 & $0.3,1.1$ & 0.10 \\
\hline
\end{tabular}

Ref., reference.

* Linear trends were assessed by assignment of ordinal variables for fish consumption tertile.

† Adjusted for maternal age at delivery, educational background, household income, occupation in early pregnancy, smoking habits, alcohol consumption, BMI before pregnancy, current history of diabetes or gestational diabetes, parity, infertility treatment, use of folic acid supplement, use of DHA and/or EPA supplements and vegetable consumption in early pregnancy.

likely to have a beneficial effect for pregnant women and women who are seeking to become pregnant.

The composite outcome, which combined oesophageal atresia, duodenal atresia, jejunoileal atresia, and/or anorectal malformation, was justified by the similar pattern of nonsignificant decreased odds for each type of atresia with an increase in fish consumption, suggesting the possibility that there is partly shared pathogenesis in this case. However, since the pathogenesis of GI tract atresia is unresolved, it is not easy to explain the association with fish consumption. The generally adverse effects of oxidative stress on organogenesis are recognised $^{(38)}$; thus, consumption of fish, in which $n-3$ PUFA are abundant, may help prevent embryonic oxidative damage $^{(24)}$. Also, $n$-3 PUFA affect insulin secretion and resistance ${ }^{(39)}$, and may possibly play a role in maintaining proper maternal glucose levels during pregnancy, and have benefits for GI tract development. There is a hypothesis that diabetes and obesity are associated with various congenital anomalies, via oxidative stress and epigenetic changes caused by hyperglycaemia ${ }^{(40,41)}$. In this study, fish consumption was inversely associated with GI tract atresia even if we restricted the participants to women without diabetes or gestational diabetes and those without obesity or overweightness; thus, maternal glucose levels may be associated with its occurrence also in pregnancies without diabetes or obesity.

Alternatively, the hedgehog family of cell signals, such as sonic hedgehog $(S h b)$, may account for the association we observed. Shb signalling is essential for development of the foregut (forming the oesophagus and proximal duodenum), midgut (forming the distal duodenum, jejunum and ileum), and hindgut (forming the rectum) ${ }^{(42)}$. In in vitro analysis of mouse cortical neuron cultures, synaptomide, a DHA metabolite, was linked to $S h b$-related gene expression ${ }^{(43)}$. Thus, $S h b$ signal regulation by the DHA in fish oil may support normal gut development. Our potential explanations do not exclude the combined occurrence of specific types of atresia. However, oesophageal atresia, small intestinal atresia and anorectal malformation sometimes occur individually; as noted, we observed an inverse association between fish consumption and isolated cases of atresia, and this probably reflects the different critical windows in the differentiation of foregut, midgut and hindgut.

Fish consumption in mid-late pregnancy also tended to be inversely associated with congenital GI tract atresia; unsurprisingly, as fish consumption in mid-late pregnancy was moderately correlated with that in early pregnancy. In the case of 
Table 4. Association of $n-3$ PUFA consumption in early pregnancy with congenital gastrointestinal tract atresia (Odds ratios and $95 \%$ confidence intervals; median values and numbers)

\begin{tabular}{|c|c|c|c|c|c|c|}
\hline & \multicolumn{5}{|c|}{ Tertile } & \multirow[b]{3}{*}{$P_{\text {for trend }}{ }^{*}$} \\
\hline & \multirow[b]{2}{*}{ Low } & \multicolumn{2}{|c|}{ Middle } & \multicolumn{2}{|c|}{ High } & \\
\hline & & OR & $95 \% \mathrm{Cl}$ & OR & $95 \% \mathrm{Cl}$ & \\
\hline \multicolumn{7}{|l|}{$n$-3 PUFA-rich fish consumption $\dagger$} \\
\hline Median intake (g/d) & 0 & $10 \cdot 7$ & & $22 \cdot 7$ & & \\
\hline No. of women & 29831 & \multirow{2}{*}{\multicolumn{2}{|c|}{$\begin{array}{c}29832 \\
24\end{array}$}} & \multirow{2}{*}{\multicolumn{2}{|c|}{$\begin{array}{c}29832 \\
18\end{array}$}} & \\
\hline No. of cases & 32 & & & & & \\
\hline Maternal age adjusted model & Ref. & 0.7 & $0.4,1.3$ & 0.5 & $0.3,1.0$ & 0.04 \\
\hline Multivariable model $\ddagger$ & Ref. & 0.7 & $0.4,1.3$ & 0.5 & $0.3,1.0$ & 0.05 \\
\hline Multivariable model $2 \S$ & Ref. & 0.9 & $0.3,1.9$ & 0.5 & $0.2,1.3$ & $0 \cdot 16$ \\
\hline \multicolumn{7}{|l|}{ Consumption of $n-3$ PUFA } \\
\hline Median intake $(\mathrm{g} / \mathrm{d})$ & 1.3 & 1.8 & & $2 \cdot 3$ & & \\
\hline No. of women & 29831 & \multicolumn{2}{|c|}{29832} & \multicolumn{2}{|c|}{29832} & \\
\hline No. of cases & 26 & \multicolumn{2}{|c|}{27} & \multicolumn{2}{|c|}{21} & \\
\hline Maternal age adjusted model & Ref. & 1.0 & $0.6,1.8$ & 0.8 & $0.4,1.4$ & 0.45 \\
\hline Multivariable modelł & Ref. & 1.0 & $0.6,1.8$ & 0.7 & $0.4,1.4$ & 0.36 \\
\hline Multivariable model $2 \S$ & Ref. & $1 \cdot 1$ & $0.5,2.5$ & 0.7 & $0 \cdot 2,1 \cdot 8$ & 0.43 \\
\hline \multicolumn{7}{|c|}{$\begin{array}{l}\text { Ref., reference. } \\
\text { * Linear trends were assessed by assignment of ordinal variables for tertile. } \\
\text { † Six fish items (salmon or trout, Japanese amberjack, sea bream, horse mackerel or sardine, saury or mackerel and eel). } \\
\text { † Adjusted for maternal age at delivery, educational background, household income, occupation in early pregnancy, smoking habits, alcohol consumption, BMI before pregnancy, } \\
\text { current history of diabetes or gestational diabetes, parity, infertility treatment, use of folic acid supplement, use of DHA and/or EPA supplements and vegetable consumption in } \\
\text { early pregnancy. } \\
\text { § Additionally adjusted for paternal age at delivery ( } n \text { 46 455). }\end{array}$} \\
\hline
\end{tabular}

jejunoileal atresia, one aetiological hypothesis is a vascular accident, such as a thromboembolic event, in mid-late pregnancy ${ }^{(8)}$. As $n-3$ PUFA are likely to reduce the risk of thrombosis $^{(21)}$, fish consumption, particularly in mid-late pregnancy, may help prevent the occurrence of jejunoileal atresia; however, due to the relatively small number of jejunoileal atresia cases and the absence of information on clinical classification of atresia, it was difficult to test this hypothesis.

The main strengths of this study are the use of a nationwide cohort of approximately 100000 mother-child pairs in Japan, and its prospective design. In regard to congenital GI tract atresia that is comparatively rare occurrence, therefore, we could clarify the temporal association between exposure and outcome. Another strength is that the JECS had information on a wide range of factors. The inverse association between fish consumption and GI tract atresia might be seen as reflecting not the beneficial effects of fish and related nutrients but rather a link with healthy maternal behaviour and socioeconomic status, because women in the high fish consumption category were likely to be never smokers and more educated, and to have higher income and vegetable intake. In this study, however, we could demonstrate the association without confounding by these factors. Also, the study gave us the opportunity to exclude participants with severe morning sickness, and to adjust for paternal age among the sub-population of the overall cohort.

Among the limitations of the study, first, the number of GI tract atresia cases was small, so it was difficult to remove the possibility of a chance finding despite our careful statistical analysis. Second, the first FFQ instructed to answer usual dietary intake during the previous year, in which are filled at around 15 weeks of gestation, was used as a marker of dietary intake in the embryonic period; that is, we did not directly assess fish intake in our target period. Third, the FFQ we used had measurement error due to self-reporting, and was not validated specifically for pregnant women, though the estimated fish intake in our population was similar to that estimated in the national survey $($ median $=32 \cdot 2 \mathrm{~g} / \mathrm{d}$ for women aged 20-29 years and $36.4 \mathrm{~g} / \mathrm{d}$ for women aged $30-39$ years $)^{(44)}$. However, the measurement errors in the study were not associated with outcome, so we considered that the point estimates of the OR would be attenuated toward the null. Fourth, the non-case group for congenital GI tract atresia may have included a small number of cases that were not diagnosed until after the first month after birth. Finally, we considered the impact of including only women who delivered a live birth. Fish and $n-3$ PUFA consumption seems unlikely to be associated with stillbirth $^{(45)}$. The majority of GI tract atresia among stillbirths would be caused by genetic and/or chromosomal factors. Therefore, it is unlikely that the examined association was distorted because we restricted to live births.

In conclusion, our results indicated fish consumption in early pregnancy may be a preventive factor for congenital GI tract atresia. Further studies may aid in achieving primary prevention of GI tract atresia, which is a major neonatal surgical disease.

\section{Acknowledgements}

The authors would like to express their gratitude to all of the JECS study participants and participating co-operating health care providers.

The JECS was funded by the Ministry of the Environment, Japan. The funders had no role in study design, data 
collection and analysis, decision to publish or preparation of the manuscript. The findings and conclusions of this article are solely the responsibility of the authors and do not represent the official views of the Ministry of the Environment, Japan.

T. M. contributed to the data collection, conducted the data analysis and drafted the manuscript. S. Y. contributed to the statistical analyses and interpretation of the results. M. O. contributed to the data collection and provided statistical expertise. T. Ku., as a paediatric surgeon, provided clinical expertise and contributed to interpretations. S. F. N., E. S., T. I., K. T. and J. Y. contributed to the data collection and interpretation of the results. M. I.-S. and Y. K. provided comments and significant consultation regarding environmental contaminants. T. Ka. and H. N. contributed to the design of the JECS, the data collection and interpretation of the results. All authors contributed to the critical revision of the manuscript and approved the final version of the manuscript

The authors declare that there are no conflicts of interest.

\section{Supplementary material}

For supplementary material/s referred to in this article, please visit https://doi.org/10.1017/S0007114518002842

\section{References}

1. International Centre on Birth Defects (2014) International Clearinghouse Centre for Birth Defects Surveillance and Research Annual Report 2014. http://www.icbdsr.org/resources/ annual-report/ (accessed April 2018).

2. Yagi M, Kohno M, Asagiri K, et al. (2015) Twenty-year trends in neonatal surgery based on a nationwide Japanese surveillance program. Pediatr Surg Int 31, 955-962.

3. Tennant PWG, Pearce MS, Bythell M, et al. (2010) 20-year survival of children born with congenital anomalies: a population-based study. Lancet 375, 649-656.

4. Stollman TH, de Blaauw I, Wijnen MH, et al. (2009) Decreased mortality but increased morbidity in neonates with jejunoileal atresia; a study of 114 cases over a 34-year period. J Pediatr Surg 44, 217-221.

5. Koivusalo AI, Sistonen SJ, Lindahl HG, et al. (2017) Long-term outcomes of oesophageal atresia without or with proximal tracheooesophageal fistula - gross types A and B. J Pediatr Surg 52, 1571-1575.

6. Rigueros Springford L, Connor MJ, Jones K, et al. (2016) Prevalence of active long-term problems in patients with anorectal malformations: a systematic review. Dis Colon Rectum 59, 570580.

7. Smith N (2014) Oesophageal atresia and tracheo-oesophageal fistula. Early Hum Dev 90, 947-950.

8. Nichol PF, Reeder A \& Botham R (2011) Humans, mice, and mechanisms of intestinal atresias: a window into understanding early intestinal development. J Gastrointest Surg $\mathbf{1 5}$, 694-700.

9. Gupta A, Bischoff A, Penñ A, et al. (2014) The great divide: septation and malformation of the cloaca, and its implications for surgeons. Pediatr Surg Int 30, 1089-1095.

10. de Jong EM, Felix JF, de Klein A, et al. (2010) Etiology of esophageal atresia and tracheoesophageal fistula: "mind the gap". Curr Gastroenterol Rep 12, 215-222.
11. Wang C, Li L \& Cheng W (2015) Anorectal malformation: the etiological factors. Pediatr Surg Int 31, 795-804.

12. Viswanathan M, Treiman KA, Kish-Doto J, et al. (2017) Folic acid supplementation for the prevention of neural tube defects: an updated evidence report and systematic review for the US Preventive Services Task Force. JAMA 317, 190-203.

13. Oddsberg J, Lu Y \& Lagergren J (2010) Maternal diabetes and risk of esophageal atresia. J Pediatr Surg 45, 2004-2008.

14. Correa A, Gilboa SM, Besser LM, et al. (2008) Diabetes mellitus and birth defects. Am J Obstet Gynecol 199, 237.e1-237.e9.

15. Block SR, Watkins SM, Salemi JL, et al. (2013) Maternal prepregnancy body mass index and risk of selected birth defects: evidence of a dose-response relationship. Paediatr Perinat Epidemiol 27, 521-531.

16. Stothard KJ, Tennant PW, Bell R, et al. (2009) Maternal overweight and obesity and the risk of congenital anomalies: a systematic review and meta-analysis. JAMA 301, 636-650.

17. Persson M, Cnattingius S, Villamor E, et al. (2017) Risk of major congenital malformations in relation to maternal overweight and obesity severity: cohort study of 1.2 million singletons. BMJ 357, j2563.

18. Waller DK, Shaw GM, Rasmussen SA, et al. (2007) Prepregnancy obesity as a risk factor for structural birth defects. Arch Pediatr Adolesc Med 161, 745-750.

19. Åberg A, Westbom L \& Källén B (2001) Congenital malformations among infants whose mothers had gestational diabetes or preexisting diabetes. Early Hum Dev 61, 85-95.

20. Gallo LA, Barrett HL \& Dekker Nitert M (2017) Review: placental transport and metabolism of energy substrates in maternal obesity and diabetes. Placenta 54, 59-67.

21. Lorente-Cebrián S, Costa AG, Navas-Carretero S, et al. (2013) Role of omega-3 fatty acids in obesity, metabolic syndrome, and cardiovascular diseases: a review of the evidence. J Physiol Biochem 69, 633-651.

22. Jones ML, Mark PJ \& Waddell BJ (2014) Maternal dietary omega-3 fatty acids and placental function. Reproduction $\mathbf{1 4 7}$, R143-R152.

23. Calder PC, Ahluwalia N, Brouns F, et al. (2011) Dietary factors and low-grade inflammation in relation to overweight and obesity. Br J Nutr 106, Suppl. 3, S5-S78.

24. Mozaffarian D \& Wu JH (2012) (n-3) Fatty acids and cardiovascular health: are effects of EPA and DHA shared or complementary? J Nutr 142, 614S-625S.

25. Kawamoto T, Nitta H, Murata K, et al. (2014) Rationale and study design of the Japan Environment and Children's Study (JECS). BMC Public Health 14, 25.

26. Michikawa T, Nitta H, Nakayama SF, et al. (2018) Baseline profile of participants in the Japan Environment and Children's Study (JECS). J Epidemiol 28, 99-104.

27. Adams SD \& Stanton MP (2014) Malrotation and intestinal atresias. Early Hum Dev 90, 921-925.

28. Yokoyama Y, Takachi R, Ishihara J, et al. (2016) Validity of short and long self-administered food frequency questionnaires in ranking dietary intake in middle-aged and elderly Japanese in the Japan Public Health Center-Based Prospective Study for the Next Generation (JPHC-NEXT) protocol area. J Epidemiol 26, 420-432.

29. Report of the Subdivision on Resources the Council for Science and Technology Ministry of Education Culture, Sports, Science and Technology, Japan (2005) Standard Tables of Food Composition in Japan, Fifth Revised Edition, 2005 Fatty Acids Section. Tokyo: National Printing Bureau (in Japanese).

30. Boulet SL, Kirby RS, Reefhuis J, et al. (2016) Assisted reproductive technology and birth defects among liveborn infants in Florida, Massachusetts, and Michigan, 2000-2010. JAMA Pediatr 170, e154934. 
31. Botto LD, Olney RS \& Erickson JD (2004) Vitamin supplements and the risk for congenital anomalies other than neural tube defects. Am J Med Genet C Semin Med Genet 125C, 12-21.

32. Green RF, Devine O, Crider KS, et al. (2010) Association of paternal age and risk for major congenital anomalies from the National Birth Defects Prevention Study, 1997 to 2004. Ann Epidemiol 20, 241-249.

33. Parker SE, Mai CT, Canfield MA, et al. (2010) Updated national birth prevalence estimates for selected birth defects in the United States, 2004-2006. Birth Defects Res A Clin Mol Teratol 88, 1008-1016.

34. WHO/CDC/ICBDSR (2014) Birth Defects Surveillance: A Manual for Programme Managers. Geneva: World Health Organization.

35. Japan Ministry of Health, Labour and Welfare (2010) Mercury included in fish and shellfish. http://www.mhlw.go.jp/topics/ bukyoku/iyaku/syoku-anzen/suigin/ (accessed April 2018).

36. US Food and Drug Administration (2017) Advice about eating fish, from the Environmental Protection Agency and Food and Drug Administration; revised fish advice. https://www.federal register.gov/documents/2017/01/19/2017-01073/advice-abouteating-fish-from-the-environmental-protection-agency-and-foodand-drug-administration (accessed April 2018).

37. Japan Ministry of Health, Labour and Welfare (2009) Dietary reference intakes for Japanese (2010). http://www.mhlw.go.jp/ stf/seisakunitsuite/bunya/kenkou_iryou/kenkou/eiyou/syokuji_ kijyun.html (accessed April 2018).

38. Wells PG, McCallum GP, Chen CS, et al. (2009) Oxidative stress in developmental origins of disease: teratogenesis, neurodevelopmental deficits, and cancer. Toxicol Sci 108, 4-18.

39. Bhaswant M, Poudyal H \& Brown L (2015) Mechanisms of enhanced insulin secretion and sensitivity with $n-3$ unsaturated fatty acids. J Nutr Biochem 26, 571-584.

40. Gabbay-Benziv R, Reece EA, Wang F, et al. (2015) Birth defects in pregestational diabetes: defect range, glycemic threshold and pathogenesis. World J Diabetes 6 , 481-488.

41. Salbaum JM \& Kappen C (2011) Diabetic embryopathy: a role for the epigenome? Birth Defects Res A Clin Mol Teratol 91, $770-780$.
42. van den Brink GR (2007) Hedgehog signaling in development and homeostasis of the gastrointestinal tract. Physiol Rev 87, 1343-1375.

43. Kharebava G, Rashid MA, Lee JW, et al. (2015) N-Docosahexaenoylethanolamine regulates hedgehog signaling and promotes growth of cortical axons. Biol Open $\mathbf{4}$, 1660-1670.

44. Japan Ministry of Health, Labour and Welfare (2015) The National Health and Nutrition Survey in Japan (2013). http:// www.mhlw.go.jp/bunya/kenkou/eiyou/h25-houkoku.html (accessed April 2018).

45. Imhoff-Kunsch B, Briggs V, Goldenberg T, et al. (2012) Effect of $n-3$ long-chain polyunsaturated fatty acid intake during pregnancy on maternal, infant, and child health outcomes: a systematic review. Paediatr Perinat Epidemiol 26, Suppl. 1, 91-107.

\section{Appendix}

Members of the JECS as of 2017 (principal investigator, T. K.): Hirohisa Saito (National Centre for Child Health and Development, Tokyo, Japan), Reiko Kishi (Hokkaido University, Sapporo, Japan), Nobuo Yaegashi (Tohoku University, Sendai, Japan), Koichi Hashimoto (Fukushima Medical University, Fukushima, Japan), Chisato Mori (Chiba University, Chiba, Japan), Shuichi Ito (Yokohama City University, Yokohama, Japan), Zentaro Yamagata (University of Yamanashi, Chuo, Japan), Hidekuni Inadera (University of Toyama, Toyama, Japan), Michihiro Kamijima (Nagoya City University, Nagoya, Japan), Takeo Nakayama (Kyoto University, Kyoto, Japan), Hiroyasu Iso (Osaka University, Suita, Japan), Masayuki Shima (Hyogo College of Medicine, Nishinomiya, Japan), Yasuaki Hirooka (Tottori University, Yonago, Japan), Narufumi Suganuma (Kochi University, Nankoku, Japan), Koichi Kusuhara (University of Occupational and Environmental Health, Kitakyushu, Japan) and Takahiko Katoh (Kumamoto University, Kumamoto, Japan). 